\title{
Long-Term Follow-Up of Disability, Cognitive, and Emotional Impairments after Severe Traumatic Brain Injury
}

\author{
Britt-Marie Stålnacke $\mathbb{D}^{1},{ }^{1}$ Britt-Inger Saveman $\mathbb{D}^{2},{ }^{2}$ and Maud Stenberg ${ }^{1}$ \\ ${ }^{1}$ Department of Community Medicine and Rehabilitation, Rehabilitation Medicine, Umeå University, Umeå, Sweden \\ ${ }^{2}$ Department of Nursing, Umeå University, Umeå, Sweden \\ Correspondence should be addressed to Britt-Marie Stålnacke; britt-marie.stalnacke@umu.se
}

Received 28 March 2019; Accepted 16 August 2019; Published 27 August 2019

Guest Editor: Mary H. Kosmidis

Copyright (C) 2019 Britt-Marie Stålnacke et al. This is an open access article distributed under the Creative Commons Attribution License, which permits unrestricted use, distribution, and reproduction in any medium, provided the original work is properly cited.

\begin{abstract}
Aim. To assess the clinical course of disability, cognitive, and emotional impairments in patients with severe TBI (s-TBI) from 3 months to up to 7 years post trauma. Methods. A prospective cohort study of s-TBI in northern Sweden was conducted. Patients aged 18-65 years with acute Glasgow Coma Scale 3-8 were assessed with the Glasgow Outcome Scale Extended (GOSE), the Hospital Anxiety and Depression Scale (HADS), and the Barrow Neurological Institute Screen for Higher Cerebral Functions (BNIS) at 3 months, 1 year, and 7 years after the injury. Results. The scores on both GOSE and BNIS improved significantly from 3 months (GOSE mean: $4.4 \pm 2.3$, BNIS mean: $31.5 \pm 7.0$ ) to 1 year (GOSE mean: $5.5 \pm 2.7, p=0.003$, BNIS mean: $33.2 \pm$ $6.3, p=0.04$ ), but no significant improvement was found from 1 year to 7 years (GOSE mean: $4.7 \pm 2.8, p=0.13$, BNIS mean: $33.5 \pm 3.9, p=0.424)$ after the injury. The BNIS subscale "speech/language" at 1 year was significantly associated with favourable outcomes on the GOSE at 7 years $(\mathrm{OR}=2.115, \mathrm{CI}: 1.004-4.456, p=0.049)$. Conclusions. These findings indicate that disability and cognition seem to improve over time after s-TBI and appear to be relatively stable from 1 year to 7 years. Since cognitive function on some of the BNIS subscales was associated with outcome on the GOSE, these results indicate that both screening and follow-up of cognitive function could be of importance for the rehabilitation of persons with s-TBI.
\end{abstract}

\section{Introduction}

Traumatic brain injury (TBI) is a major public health problem all over the world and is a leading cause of death and long-term disability in people of working age [1]. The most widely used TBI classification is based on the level of consciousness (LOC) on admission, defined by the Glasgow Coma Scale (GCS) [2]. The annual incidence of all levels of traumatic brain injury (TBI; mild, moderate, and severe according to GCS 3-15) is 250-350,000/year [3-5]. Severe traumatic brain injury (s-TBI) varies between GCS of 3 and 8 on admission; is much rarer, with incidence estimates of $3-12 / 100,000 /$ year [4]; and has the highest mortality rate [6]. The damage constitutes a major health problem and causes great suffering for both patients and their families with extensive consequences both at the individual and the community level. Patients with s-TBI comprise a heterogeneous group with deficits and impairments of varying complexity that can affect both the physical and mental status as well as social and family life [7]. Cognitive deficits and emotional impairments including symptoms of depression and anxiety have been shown to affect the achievement of rehabilitation goals after TBI and are a major cause of disability after TBI [8]. Since cognitive and emotional impairments are not noticeable initially, it is difficult for other people to understand the severity and problems a person with s-TBI is having to cope with.

Various scales have been developed for the assessment of cognition, depression, anxiety, and disability after TBI. Most studies of cognitive deficits have focused on deficits in memory, attention/concentration, and visuospatial abilities $[9,10]$, but the impact on a combination of these areas together with affective functions, orientation, and awareness has not been studied to the same extent. The Barrow Neurological Institute Screen for Higher Cerebral Functions (BNIS) is an instrument that has been used to measure both 
cognitive and affective disturbances in patients with TBI [11]. The prevalence of depression and anxiety after TBI has been shown to vary widely and may be related to different methods of assessment. One of the most common instruments used in clinical healthcare is the Hospital Anxiety and Depression Scale (HADS) [12] that has been studied in a large number of different patient groups. To measure outcome after sTBI, the Glasgow Outcome Scale (GOS) is commonly used $[13,14]$. This is a global and somewhat crude instrument that only roughly discriminates different levels of disability. A more sophisticated version of the GOS, the Glasgow Outcome Scale Extended (GOSE), has thus been developed, allowing for a more fine-tuned categorisation of posttraumatic disability.

Most studies of s-TBI focus on the acute phase and then a short follow-up period. In a previous national multicentre study of patients with s-TBI (the ProBrain study), we assessed the clinical course of cognitive and emotional function from 3 weeks to 1 year after trauma [15].

In the present study, the aim was to assess the clinical course of patients with s-TBI from northern Sweden (a subgroup of the ProBrain population) on disability, cognitive, and emotional impairments up to 7 years after trauma.

\section{Methods}

2.1. Design. The design of the study is a prospective cohort study.

2.2. Patients. Inclusion criteria for the study were patients aged 18-65 years, with acute s-TBI with the lowest nonsedated GCS (Glasgow Coma Scale) [2] score 8 of 3-8 within 24 hours post trauma. The exclusion criterion for this study was death within 3 weeks of injury. Thirty-seven patients (11 female, 26 male) with s-TBI were admitted from January 2010 to December 2011 to the Neurotrauma Center (NC) at Umeå University Hospital. These 37 patients were included as part of the Swedish-Icelandic multicentre ProBrain study, a prospective 2-year study with a one-year follow-up of severe traumatic brain injuries [15].

2.3. Procedure. The 37 patients with s-TBI were treated in the acute phase at the Neurotrauma Center (NC) at Umeå University Hospital, covering the North Health Region (NHR) which covers a geographical area which comprises almost half of the total area of Sweden $\left(136,373 \mathrm{~km}^{2}\right)$. It is divided into four counties and has approximately 900,000 inhabitants, thus comprising only $10 \%$ of the total national population. Patients were evaluated at 3 months, 1 year, and up to 7 years after the injury. Assessment took place in the patients' current care setting or at their homes and was performed by the same rehabilitation physician at all time points.

\subsection{Instruments}

2.4.1. The Barrow Neurological Institute Screen for Higher Cerebral Functions (BNIS). The Barrow Neurological Institute Screen for Higher Cerebral Functions (BNIS) [11] is a cognitive screening test for speech and language func- tions, orientation, attention/concentration, visuospatial and visual problem solving, memory, affect, and awareness. BNIS yields a total score and seven subscale scores. The total score (maximum 50 points) reflects overall cognitive function and consists of the results from a prescreen plus the 7 subscale scores (speech and language, 15 points; orientation, 3 points; attention/concentration, 3 points; visual and visuospatial problem solving, 8 points; memory, 7 points; affect, 4 points; and awareness, 1 point). A total subscale score can be obtained. Higher scores reflect a higher level of functioning. If the total BNIS score is below 47 points, further cognitive investigation is recommended. The BNIS has been validated for a Swedish population $[16,17]$.

2.4.2. The Hospital Anxiety and Depression Scale (HADS). The Hospital Anxiety and Depression Scale (HADS) [12] was used to identify the presence and degree of anxiety and depression. It consists of 14 items (7 items in each subscale, HADS-depression (HADS-D) and HADS-anxiety (HADS-A)) which are assessed on a 4-point Likert scale (range 0-3), where the total score is the sum of each subscale (range 0-21). Cut-offs for both subscales of 8 or higher were used to determine "caseness" [12]. The HADS is an established screening tool for anxiety and depression and it has been used previously in patients with TBI. The HADS has acceptable reliability, sensitivity and specificity in assessing symptom severity in anxiety and depression in various populations [18].

2.4.3. The Glasgow Outcome Scale Extended (GOSE). The Glasgow Outcome Scale Extended (GOSE) [19] extends the 5 categories of the previously developed Glasgow Outcome Scale (GOS) [19] to 8 on disability, thereby increasing its sensitivity. The 8 categories span from "death" (score 1) to "upper good recovery" (score 8). GOSE was also dichotomised into "unfavourable outcome" (GOSE 1-4), and "favourable outcome" (GOSE 5-8). The GOSE has good interrater reliability [19] and validity [20] and is an established measure of global outcome after traumatic brain injury.

\section{Statistical Analysis}

Data were analysed with SPSS, version 25, for Windows. Data were reported as frequencies or median and means. Nonparametric tests were used as the samples were small and/or not normally distributed. Thus, Wilcoxon's signedrank test was used for the study of paired observation variables. The level of statistical significance was set as $p$ $=0.05$. Univariate binary logistic regression analyses were performed to explore associations between the following variables at 1 year: BNIS (total score and the 7 subscale scores), HADS-A, and HADS-D with GOSE as a dependent variable at follow-up after 7 years ("unfavourable and favourable outcomes"). Variables that had a $p$ value $<0.1$ in the univariate regression analyses were then included in a multivariate model using a forward method. The results of the logistic regression analyses are presented as an odds ratio (OR). The reliability of the OR is expressed 
as a $95 \%$ confidence interval (CI). Statistical significance was set at $p<0.05$ for the multivariate regression analysis.

\section{Ethical Considerations}

The study has been approved by the Research Ethical Review Board Umeå Sweden, D-nr. 2016/444-31.

\section{Results}

At the time of follow-up, 9 participants were deceased. Six of them ( 4 men and 2 women) had died within the first year and three later on. GCS 3 was seen in 9 of the included patients and hospital deaths occurred in 4 of these patients. Two of these patients died at the NC due to respiratory complications, and traumatic and inoperable intracranial aneurysm, respectively. One of the fatalities suffered from multiple illnesses at the time of injury, two of the patients with very severe brain injury (GCS 3) died because of respiratory infection/infection/pneumonia complications, and one patient died from intracerebral bleeding after transportation from NC to the local hospital. Patients with hospital death had a significantly higher mean age in comparison with patients who survived $(52.8 \pm 17.8$ vs. $41.3 \pm 15.1, p=0.048)$.

Thus, there were 28 remaining patients with s-TBI who were eligible to participate in this study. Three participants could not be identified, and four patients declined to participate. Therefore, 21 persons were included in the 7 years follow-up (14 men and 7 women). They were aged between 17 and 64 years at the time of the injury (mean 41 years). Their age varied from 27 to 70 years (median 49) at the time of the follow-up. The median for the lowest nonsedated GCS within 24 hours post trauma was GCS 5 (3-8). Causes of the injury were falls in 9 cases and traffic incidents in 8 cases (Table 1). The participants with s-TBI were hospitalised on average 62 (16-250) days and in a specialised rehabilitation department for an average of 35 (0-127) days.

For background information, see Tables 1 and 2. The number of part-time/full-time workers or being a student had decreased from 17 at time for injury to 10 after 7 years, and participants on full or partial sick leave had increased from 3 to 9. Four participants had personal assistants 24/7 or social care several times daily at follow-up. The ability to keep or get a driving license had decreased from 16 to 13 of the injured participants (Table 2). Eight of the participants with s-TBI had returned to earlier employment or retired. One of the participants with s-TBI also had an incomplete traumatic spinal cord injury but could walk at follow-up.

5.1. GOSE. GOSE improved significantly from 3 months (median $4.5(1-8)$, mean: $4.4 \pm 2.3$ ) to 1 year (median 7 (1-8), mean: $5.5 \pm 2.7 ; p=0.003$ ) and close to significantly from 3 months and 7 years (median 5 (1-8), mean: 4.7 $\pm 2.8 ; p=0.059)$. No significant improvement was found on GOSE from 1 year to 7 years $(p=0.137)$. At 3 months, GOSE $1-4$ was seen in $50 \%$ and GOSE $5-8$ in $50 \%$. At 1 year, GOSE 1-4 was seen in $36 \%$ and GOSE $5-8$ in $64 \%$. At 7 years, GOSE 1-4 was seen in $42 \%$ and GOSE 5-8
TABLE 1: Demographic injury characteristics for persons with severe traumatic brain injury in northern Sweden (approximately 900,000 inhabitants).

\begin{tabular}{lcc}
\hline & $N$ & $\%$ \\
\hline Included year: 2010-2011 & 37 & 100 \\
Included at follow-up & 21 & 57 \\
Age at follow-up $(n=21)$ & & \\
$\quad$ Median $49(27-70)$ years & & \\
Gender $(n=21)$ & & \\
$\quad$ Male & 14 & 66 \\
Female & 7 & 33 \\
Diagnosis $(n=21)$ & & \\
$\quad$ S062 diffuse brain injury & 6 & 29 \\
S063 focal brain injury & 2 & 10 \\
S064 EPH & 4 & 19 \\
S065 traumatic SDH & 7 & 33 \\
S066 traumatic SAH & 1 & 5 \\
S068 other & 1 & 5 \\
Influence of alcohol misuse at time of injury & 18 & \\
Cause of injury $(n=21)$ & 9 & 43 \\
Fall & 8 & 38 \\
Traffic & 3 & 14 \\
Sport & 1 & 5 \\
Unknown & & \\
Education $(n=21)$ & & \\
$<12$ years & & \\
12 years & & \\
$>12$ year & & 38 \\
\hline
\end{tabular}

EPH: epidural hematoma; SDH: subdural hematoma; SAH: subarachnoidal hematoma.

in 58\%. Good recovery (GOSE 7-8) was seen in 59\%, both at 1 year and at 7 years follow-up (see Table 3 ).

5.2. Hospital Anxiety and Depression Scale-Depression (HADS-D) Scores. No statistically significant differences were found for HADS-D from 3 months (median: 2 (010), mean: $3.1 \pm 3.0)$ to 1 year (median: $2.5(0-12)$, mean: $3.4 \pm 2.5 ; p=0.793$ ), from 3 months to 7 years (median: 3 (0-11), mean: $3.6 \pm 3.0 ; p=0.875)$, and from 1 year to 7 years $(p=0.391)$. HADS-D scores above cut-off occurred in 3 participants at 3 months, in 5 participants at 1 year, and in 3 participants at 7 years after injury (see Table 3 ).

5.3. Hospital Anxiety and Depression Scale-Anxiety (HADSA) Scores. There was a close to significant difference between the scores of HADS-A from 3 months (median: 3.5 (0-14), mean: $4.5 \pm 3.8)$ to 1 year (median: $4(0-12)$, mean: $3.8 \pm$ $3.5 ; p=0.059$ ), but not from 3 months to 7 years (median: 3 (0-13), mean: $3.7 \pm 3.7 ; p=0.373$ ) and not from 1 year to 7 years $(p=0.699)$. HADS-A scores above cut-off occurred in 5 participants at 3 months, in 4 participants at 1 year, and in 3 participants at 7 years after injury (see Table 3 ). 
TABLE 2: Marital status, employment and livelihood, personal support, driving license at time of injury (2010-2011) and at follow-up after 7 years $(n=21)$.

\begin{tabular}{|c|c|c|c|c|}
\hline & \multicolumn{2}{|c|}{ Time of injury } & \multicolumn{2}{|c|}{$\begin{array}{c}\text { Follow-up after } 7 \\
\text { years }\end{array}$} \\
\hline & $n$ & $\%$ & $n$ & $\%$ \\
\hline \multicolumn{5}{|l|}{ Marital status } \\
\hline Unmarried without underage children living at home & 4 & 19 & 4 & 19 \\
\hline Unmarried with underage children living at home & 1 & 5 & 0 & 0 \\
\hline Married/cohabitation without underage children living at home & 9 & 43 & 10 & 48 \\
\hline Married/cohabitation with underage children living at home & 6 & 29 & 5 & 24 \\
\hline Married/cohabitation with grown child living at home & 1 & 5 & 1 & 5 \\
\hline \multicolumn{5}{|l|}{ Employment } \\
\hline Working or studying part time or full time & 17 & 81 & 10 & 48 \\
\hline Sick leave full time or part time & 3 & 14 & 9 & 43 \\
\hline Social care & 1 & 5 & 1 & 5 \\
\hline Other & 0 & 0 & 1 & 5 \\
\hline \multicolumn{5}{|l|}{ Personal support } \\
\hline Personal assistant $24 / 7$ & 0 & 0 & 2 & 10 \\
\hline Social care several times daily & 0 & 0 & 2 & 10 \\
\hline Driving license & 16 & 76 & 13 & 62 \\
\hline
\end{tabular}

TABLe 3: HADS-depression (HADS-D), HADS-anxiety (HADS-A), and GOSE scores at 3 months, 1 year, and 7 years.

\begin{tabular}{|c|c|c|c|c|c|c|}
\hline & 3 months & 1 year & $\begin{array}{c}3 \text { months versus } 1 \text { year ( } p \\
\text { value) }\end{array}$ & 7 years & $\begin{array}{c}3 \text { months versus } 7 \text { years ( } p \\
\text { value) }\end{array}$ & $\begin{array}{c}1 \text { year versus } 7 \text { years }(p \\
\text { value })\end{array}$ \\
\hline $\begin{array}{l}\text { HADS-D total score median } \\
\text { (range) }\end{array}$ & $2(0-10)$ & $\begin{array}{l}2.5(0- \\
12)\end{array}$ & 0.793 & $\begin{array}{l}3.0(0- \\
11)\end{array}$ & 0.875 & 0.391 \\
\hline HADS-D mean (SD) & $3.1(3.0)$ & $\begin{array}{c}3.4 \\
(2.5)\end{array}$ & & $\begin{array}{c}3.6 \\
(3.0)\end{array}$ & & \\
\hline $\begin{array}{l}\text { HADS-A total score median } \\
\text { (range) }\end{array}$ & $\begin{array}{l}3.5(0- \\
14)\end{array}$ & $\begin{array}{c}4(0- \\
12)\end{array}$ & 0.059 & $\begin{array}{c}3.0(0- \\
13)\end{array}$ & 0.373 & 0.699 \\
\hline HADS-A mean (SD) & $4.5(3.8)$ & $\begin{array}{c}3.8 \\
(3.5)\end{array}$ & & $\begin{array}{c}3.7 \\
(3.7)\end{array}$ & & \\
\hline$H A D S-D \geq 8 \%(n)$ & $12.0(3)$ & $17.9(5)$ & 0.694 & $8.1(3)$ & & 0.442 \\
\hline$H A D S-A \geq 8 \%(n)$ & $19.2(5)$ & $14.3(4)$ & 0.085 & $15.0(3)$ & & 0.216 \\
\hline GOSE median (range) & $4.5(1-8)$ & $7(1-8)$ & 0.003 & $5(1-8)$ & 0.059 & 0.137 \\
\hline GOSE mean (SD) & $4.4(2.3)$ & $\begin{array}{c}5.5 \\
(2.7)\end{array}$ & & $\begin{array}{c}4.7 \\
(2.8)\end{array}$ & & \\
\hline GOSE $1-4(n \%)$ & 48.6 & 35.1 & 0.001 & 42 & & 0.001 \\
\hline GOSE 5-8 $(n \%)$ & 48.6 & 64.9 & & 58 & & \\
\hline
\end{tabular}

5.4. BNIS. The BNIS total subscale score improved significantly on the paired $t$-tests from 3 months to 1 year post injury $(p=0.042)$. From 1 year to 7 years, no further improvement was found.

No significant improvement on the separate BNIS subscales was shown from 3 months to 7 years after the injury (see Table 4).

5.5. Outcome as Assessed with the Glasgow Outcome Scale Extended (GOSE). In the univariate logistic regression analysis GOSE after 7 years as a dependent variable, several variables 1 year after the injury had a $p$ value $<0.1$. Associations ( $p$ value $<0.1$ ) were found between the following variables after 1 year and "favourable outcome" on the GOSE: BNIS total subscale score $(\mathrm{OR}=1.197, \mathrm{CI}$ : $0.985-1.455$, and $p=0.070)$, BNIS subscale speech/language (OR $=2.115, \mathrm{CI}$ : $1.004-4.456$, and $p=0.049)$, visuospatial and visual problem solving $(\mathrm{OR}=1.765$, CI: $0.964-3.232$, and $p=0.066)$, and memory $(\mathrm{OR}=1.772$, CI: $0.933-3.365$, and $p=0.080)$. No associations were found between the BNIS subscales orientation, affect, awareness, HADS-A, and HADS-D after 1 year and GOSE at 7 years. The three BNIS subscales 
TABLE 4: BNIS subscale scores and total BNIS scores at 3 months, 1 year, and 7 years.

\begin{tabular}{lccccc}
\hline BNIS score & $\begin{array}{c}3 \text { months }(n=25) \\
\text { median }(\text { range })\end{array}$ & $\begin{array}{c}1 \text { year }(n=28) \\
\text { median }(\text { range })\end{array}$ & $\begin{array}{c}\text { months vs. 1 year } \\
(p \text { value })\end{array}$ & $\begin{array}{c}7 \text { years }(n=13) \\
\text { median }(\text { range })\end{array}$ & $\begin{array}{c}1 \text { year vs. 7 years } \\
(p \text { value })\end{array}$ \\
\hline Speech/language & $14(10-15)$ & $14(10-15)$ & 0.398 & $15(9-15)$ & 0.713 \\
Orientation & $3(1-3)$ & $3(2-3)$ & 0.317 & $3(2-3)$ & 1.000 \\
Attention/concentration & $2(0-3)$ & $2(1-3)$ & 0.392 & $2(1-3)$ & 0.317 \\
$\begin{array}{l}\text { Visuospatial and visual } \\
\text { problem solving }\end{array}$ & $6(2-8)$ & $6(1-8)$ & 0.054 & $6(3-5)$ & 0.496 \\
Memory & $4(1-7)$ & $5(0-7)$ & 0.143 & $4(1-6)$ & 0.226 \\
Affect & $4(2-4)$ & $3(2-4)$ & 0.477 & $4(2-4)$ & 0.739 \\
Awareness & $1(0-1)$ & $1(0-1)$ & 0.705 & $0(0-1)$ & 0.257 \\
$\begin{array}{l}\text { Total BNIS subscale score } \\
\text { mean (SD) }\end{array}$ & $31.5(7.0)$ & $33.2(6.3)$ & 0.042 & $33.5(3.9)$ & 0.424 \\
\hline
\end{tabular}

speech/language, visuospatial and visual problem solving, and memory were incorporated into a multivariate model. The analysis showed that statistically significant associations were only obtained for speech and language $(\mathrm{OR}=2.115$, CI: $1004-4.456$, and $p=0.049$ ).

\section{Discussion}

This study shows that both disability measured with the GOSE and cognition with the BNIS improved significantly from 3 months to 1 year while no further improvement was shown 7 years after the injury.

Nine of the included patients were deceased at the time of follow-up. The majority (six) had died within the first year and three later on. In the group of patients that could be followed up 7 years after the severe traumatic injury, the level of recovery and function varied hugely, from two of the participants having personal assistants all day and night to ten who were working or studying part- or full time. However, the patients who died were older and had lower GCS compared with the survivors. As was the case in previous studies, the majority were males $[3,21]$. The severity of TBI on the acute GCS (median GCS) was consistent with other prospective studies of s-TBI [20]. Falls are a common cause of TBI, and in some previous Scandinavian studies, falls have been reported to cause most incidents of s-TBI $[3,21]$. Falls were also the most frequent cause of injury in our population of working aged adults. The second most common cause of injury in this study was transport accidents which in American and Australian studies is reported as being the predominant cause [22]. Indications of alcohol use at the time of injury have been shown to be a risk factor for TBI $[23,24]$. In our study, almost half were under the influence of alcohol and/or drugs at the time of injury. This is a much higher rate of alcohol use in patients with s-TBI than that reported in a Norwegian study (32\%) [25] and in previous studies of patients with $\mathrm{m}$-TBI conducted at the same university hospital as the present study [26]. These findings indicate the existence of high-risk populations and the need for preventive measures.

s-TBI is a heterogeneous group with various deficits and long-term outcomes. This was also shown in our population.
Although the patients were initially classified as severe TBI with low GCS scores [2], 1 year after the injury, the overall outcome was encouragingly favourable (GOSE 5-8, 65\%) with significantly increased scores on the GOSE. However, this positive trend did not continue over the coming years on the GOSE as the scores had decreased at 7 years. Similar results were found regarding the BNIS at 1 year with higher total scores compared with 3 months but no further improvement. These findings of GOSE recovery plateauing 1 year after the injury are in accordance with the results reported in another study [27] of patients with moderate and severe TBI examined from 3 months to 24 months after the injury. The lower GOSE scores at 7 years could be seen in the concept of TBI as a chronic health condition where factors such as age and preinjury employment may play a role for the long-term outcome [28].

There are probably several reasons for the varying longterm results in the present study. In a previous study of the same population of patients with s-TBI, we studied the clinical pathways 3 months after the severe traumatic injury in the rural area of the North Health Region that covers almost half of Sweden. Most patients were swiftly transported direct to the regional well-monitored neurosurgical and neurointensive care unit. In contrast, the postacute clinical pathways less clearly reflected an optimised medical and rehabilitative strategy. Patients were often transferred back to local hospitals at a fairly early stage [29]. Since subsequent treatment and rehabilitation interventions varied after injury, there were probably no further long-term follow-up strategies from the healthcare services for these patients.

As s-TBI comprises injuries with wide variations as regards complexity of impairments and functional outcomes, prognostic indicators are essential for rehabilitation and treatment.

In a study of prognostic factors for s-TBI after 1 year of the same study population, we used two classification systems of computed tomography (CT) of the brain in the acute phase for prognostication and a prognostic calculator protocol (Corticosteroid Randomisation after Significant Head Injury (CRASH) acute calculator protocol). Neither CT nor CRASH yielded clinically useful predictions of outcome at 1 year post injury [30]. In the present study, the BNIS subscales 
speech/language, visuospatial and visual problem solving, and memory at 1 year were significantly or close to significantly associated with "favourable outcome" on the GOSE at follow-up after 7 years. However, in the multivariate model, significant association was only obtained for speech and language. This result is in line with the previous multicentre study ProBrain which reported relationships between some of the BNIS subscales and outcome on the GOSE 1 year after the injury [15].

Although the BNIS scores improved over time in our study, the scores at 1 and 7 years were in a range that was similar to that reported by Swedish TBI patients from a neurorehabilitation clinic [17] which could indicate that the long-term results are probably relatively consistent. However, it is worth remembering that the BNIS is a screening instrument that can be used to detect patients in need of comprehensive cognitive neuropsychological assessment.

This study has several strengths, such as a prospective design and close collaboration as regards case identification, which meant it was possible for most patients to be included. In addition, the extant protocols allowed for referral of patients with a seemingly dismal prognosis. Furthermore, one of the authors (MS) examined all patients at 3 months, at 1 year, and at 7 years and ensured that data were precisely and completely documented, making the amount of missing or secondary data minimal. However, the study is limited by the relatively small study population, of s-TBI, but the subset of this severe group of TBI is rare in comparison with the great majority of mild-moderate TBI. Nevertheless, the included patients comprised a near-total regional cohort population of incident s-TBI patients injured over a period of two years. Most prior studies using the BNIS have used heterogeneous study populations with a mix of diagnoses and different TBI grades $[17,31]$ which were studied at different time points after the trauma.

A limitation of the study is that not all patients could be followed up, but only three participants could not be identified and another four declined to participate. Another limitation is the heterogeneity of the age of the participants. At follow-up 7 years after the injury, the youngest patient was 27 years old and the oldest was 70 years old. Since outcomes may differ in different age groups, this could be seen as a considerable methodological limitation. However, the study population could reflect the real-life population of persons with s-TBI initially treated at a university hospital.

\section{Conclusion}

The results indicate that cognition on the BNIS improves over time after s-TBI and appears to be relatively stable from 3 months to 1 year. Since no further improvement was seen at 7 years, the long-term results are probably relatively consistent. Cognitive function on some of the BNIS subscales was associated with outcome on the GOSE. Therefore, it seems that both screening and follow-up of cognitive function could be of importance for the rehabilitation of persons with s-TBI.

\section{Data Availability}

The datasets generated and/or analysed in this study are not publicly available as the Ethical Review Board has not approved the public availability of these data.

\section{Conflicts of Interest}

The authors declare no conflict of interest.

\section{Acknowledgments}

This study was supported by grants from the Swedish Association for Survivors of Polio, Accident and Injury.

\section{References}

[1] F. Tagliaferri, C. Compagnone, M. Korsic, F. Servadei, and J. Kraus, "A systematic review of brain injury epidemiology in Europe," Acta Neurochirurgica, vol. 148, no. 3, pp. 255268, 2006.

[2] G. Teasdale and B. Jennett, "Assessment of coma and impaired consciousness. A practical scale,” Lancet, vol. 2, no. 7872, pp. 81-84, 1974.

[3] J. Styrke, B. M. Stålnacke, P. Sojka, and U. Björnstig, "Traumatic brain injures in a well-defined population: epidemiological aspects and severity," Journal of Neurotrauma, vol. 24, no. 9, pp. 1425-1436, 2007.

[4] S. Kleiven, P. M. Peloso, and H. Holst, "The epidemiology of head injuries in Sweden from 1987 to 2000," Injury Control and Safety Promotion, vol. 10, no. 3, pp. 173-180, 2003.

[5] R. Nguyen, K. M. Fiest, J. McChesney et al., "The international incidence of traumatic brain injury: a systematic review and meta-analysis," The Canadian Journal of Neurological Sciences, vol. 43, no. 6, pp. 774-785, 2016.

[6] M. Maegele, R. Lefering, O. Sakowitz et al., "The incidence and management of moderate to severe head injury," Deutsches Ärzteblatt International, vol. 116, no. 10, pp. 167-173, 2019.

[7] A. C. Hale, K. M. Bohnert, R. Grekin, and R. K. Sripada, "Traumatic brain injury in the general population," The Journal of Nervous and Mental Disease, vol. 207, no. 1, pp. 38-42, 2019.

[8] C. Bornhofen and S. McDonald, "Emotion perception deficits following traumatic brain injury: a review of the evidence and rationale for intervention," Journal of the International Neuropsychological Society, vol. 14, no. 4, pp. 511-525, 2008.

[9] S. Sigurdardottir, N. Andelic, E. Wehling et al., "Neuropsychological functioning in a national cohort of severe traumatic brain injury," The Journal of Head Trauma Rehabilitation, vol. 30, no. 2, pp. E1-12, 2015.

[10] W. H. A. Ryu, N. K. Cullen, and M. T. Bayley, "Early neuropsychological tests as correlates of productivity 1 year after traumatic brain injury: a preliminary matched case-control study," International Journal of Rehabilitation Research, vol. 33, no. 1, pp. 84-87, 2010.

[11] G. P. Prigatano and J. L. Wong, "Cognitive and affective improvement in brain dysfunctional patients who achieve inpatient rehabilitation goals," Archives of Physical Medicine and Rehabilitation, vol. 80, no. 1, pp. 77-84, 1999.

[12] A. S. Zigmond and R. P. Snaith, "The hospital anxiety and depression scale," Acta Psychiatrica Scandinavica, vol. 67, no. 6, pp. 361-370, 1983. 
[13] B. Jennett, "Assessment of outcome after severe brain damage - a practical scale," Lancet, vol. 305, no. 7905, pp. 480484, 1975.

[14] J. T. King Jr., P. M. Carlier, and D. W. Marion, "Early Glasgow Outcome Scale scores predict long-term functional outcome in patients with severe traumatic brain injury," Journal of Neurotrauma, vol. 22, no. 9, pp. 947-954, 2005.

[15] M. Stenberg, A. K. Godbolt, C. Nygren de Boussard, R. Levi, and B. M. Stålnacke, "Cognitive impairment after severe traumatic brain injury, clinical course and impact on outcome: a Swedish-Icelandic study," Behavioural Neurology, vol. 2015, Article ID 680308, 12 pages, 2015.

[16] V. Denvall, S. Elmstahl, and G. P. Prigatano, "Replication and construct validation of the Barrow Neurological Institute Screen for Higher Cerebral Function with a Swedish population," Journal of Rehabilitation Medicine, vol. 34, no. 4, pp. 153-157, 2002.

[17] C. Hofgren, E. Esbjornsson, H. Aniansson, and K. S. Sunnerhagen, "Application and validation of the Barrow Neurological Institute Screen for Higher Cerebral Functions in a control population and in patient groups commonly seen in neurorehabilitation," Journal of Rehabilitation Medicine, vol. 39, no. 7, pp. 547-553, 2007.

[18] I. Bjelland, A. A. Dahl, T. T. Haug, and D. Neckelmann, "The validity of the Hospital Anxiety and Depression Scale," Journal of Psychosomatic Research, vol. 52, no. 2, pp. 69-77, 2002.

[19] J. T. L. Wilson, L. E. L. Pettigrew, and G. M. Teasdale, "Structured interviews for the Glasgow Outcome Scale and the Extended Glasgow Outcome Scale: guidelines for their use," Journal of Neurotrauma, vol. 15, no. 8, pp. 573-585, 1998.

[20] H. S. Levin, C. Boake, J. Song et al., "Validity and sensitivity to change of the Extended Glasgow Outcome Scale in mild to moderate traumatic brain injury," Journal of Neurotrauma, vol. 18, no. 6, pp. 575-584, 2001.

[21] N. Andelic, A. Anke, T. Skandsen et al., "Incidence of hospitaladmitted severe traumatic brain injury and in-hospital fatality in Norway: a national cohort study," Neuroepidemiology, vol. 38, no. 4, pp. 259-267, 2012.

[22] P. G. Harradine, J. B. Winstanley, R. Tate, I. D. Cameron, I. J. Baguley, and R. D. Harris, "Severe traumatic brain injury in New South Wales: comparable outcomes for rural and urban residents," The Medical Journal of Australia, vol. 181, no. 3, pp. 130-134, 2004.

[23] R. C. Opreanu, D. Kuhn, and M. D. Basson, "Influence of alcohol on mortality in traumatic brain injury," Journal of the American College of Surgeons, vol. 210, no. 6, pp. 9971007, 2010.

[24] C. M. Chen, H. Y. Yi, Y. H. Yoon, and C. Dong, "Alcohol Use at Time of Injury and Survival Following Traumatic Brain Injury: Results From the National Trauma Data Bank," Journal of Studies on Alcohol and Drugs, vol. 73, no. 4, pp. 531541, 2012.

[25] N. Andelic, E. Bautz-Holter, P. Ronning et al., "Does an early onset and continuous chain of rehabilitation improve the long-term functional outcome of patients with severe traumatic brain injury?," Journal of Neurotrauma, vol. 29, no. 1, pp. 66-74, 2012.

[26] B. M. Stålnacke, U. Björnstig, K. Karlsson, and P. Sojka, “Oneyear follow-up of mild traumatic brain injury: post-concussion symptoms, disabilities and life satisfaction in relation to serum levels of S-100B and neurone-specific enolase in acute phase,"
Journal of Rehabilitation Medicine, vol. 37, no. 5, pp. 300-305, 2005.

[27] M. Sandhaug, N. Andelic, B. Langhammer, and A. Mygland, "Functional level during the first 2 years after moderate and severe traumatic brain injury," Brain Injury, vol. 29, no. 12, pp. 1431-1438, 2015.

[28] M. V. Forslund, P. B. Perrin, C. Røe et al., "Global outcome trajectories up to 10 years after moderate to severe traumatic brain injury," Frontiers in Neurology, vol. 10, p. 219, 2019.

[29] M. Stenberg, L. O. Koskinen, R. Levi, and B. M. Stålnacke, "Severe traumatic brain injuries in Northern Sweden: a prospective 2-year study," Journal of Rehabilitation Medicine, vol. 45, no. 8, pp. 792-800, 2013.

[30] M. Stenberg, L. O. D. Koskinen, P. Jonasson, R. Levi, and B. M. Stålnacke, "Computed tomography and clinical outcome in patients with severe traumatic brain injury," Brain Injury, vol. 31, no. 3, pp. 351-358, 2017.

[31] S. R. Borgaro, G. P. Prigatano, C. Kwasnica, S. Alcott, and N. Cutter, "Disturbances in affective communication following brain injury," Brain Injury, vol. 18, no. 1, pp. 33-39, 2004. 


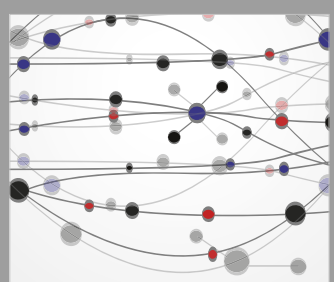

The Scientific World Journal
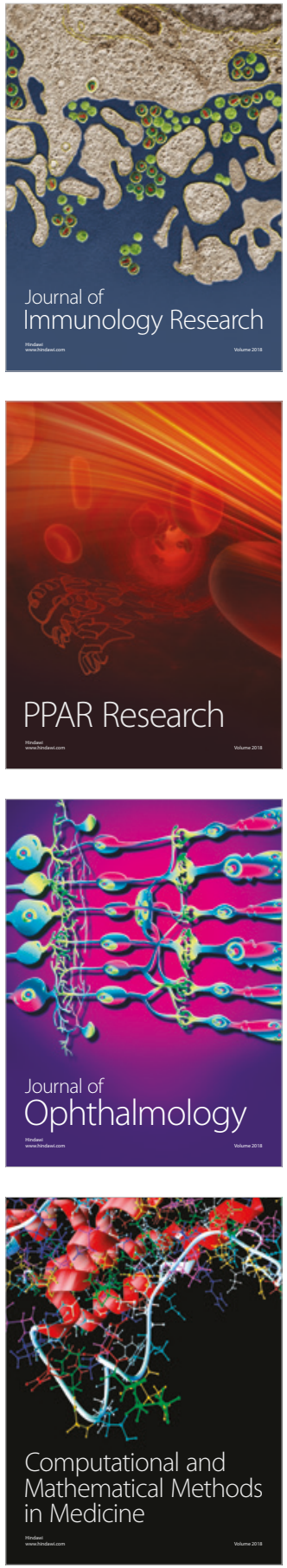

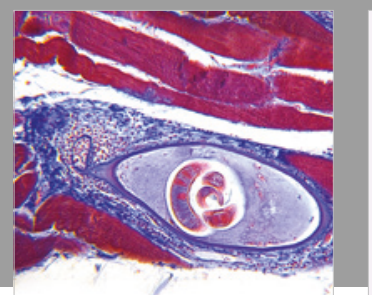

Gastroenterology Research and Practice

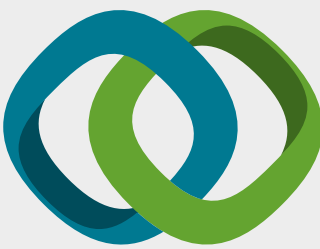

\section{Hindawi}

Submit your manuscripts at

www.hindawi.com
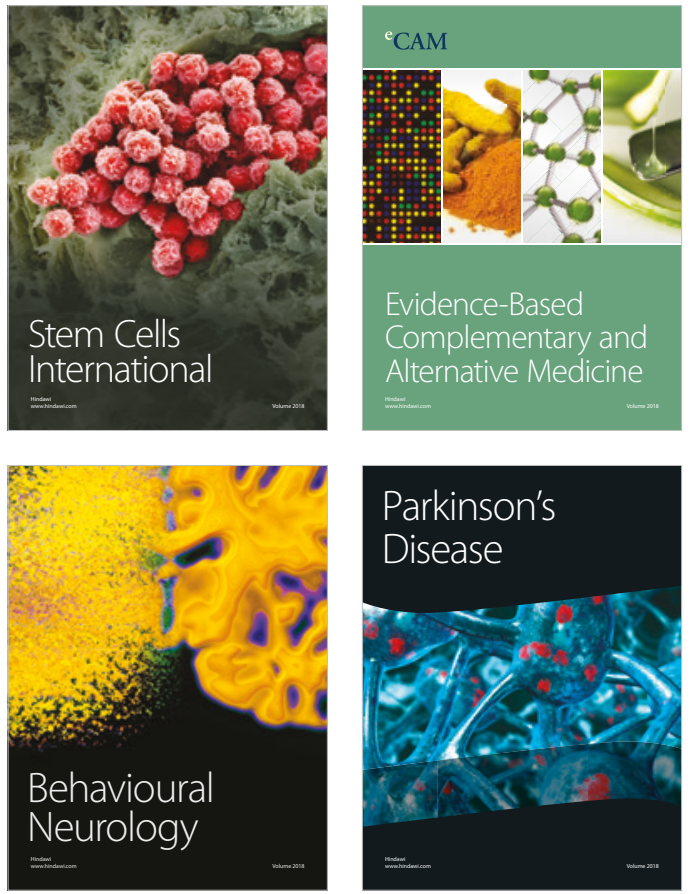

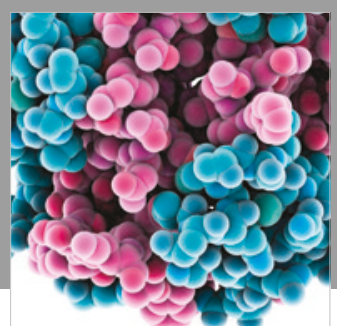

ournal of

Diabetes Research

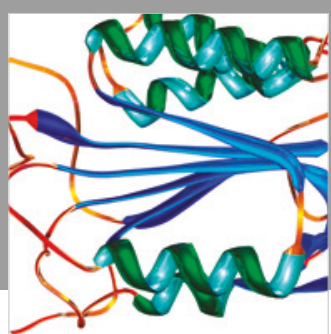

Disease Markers
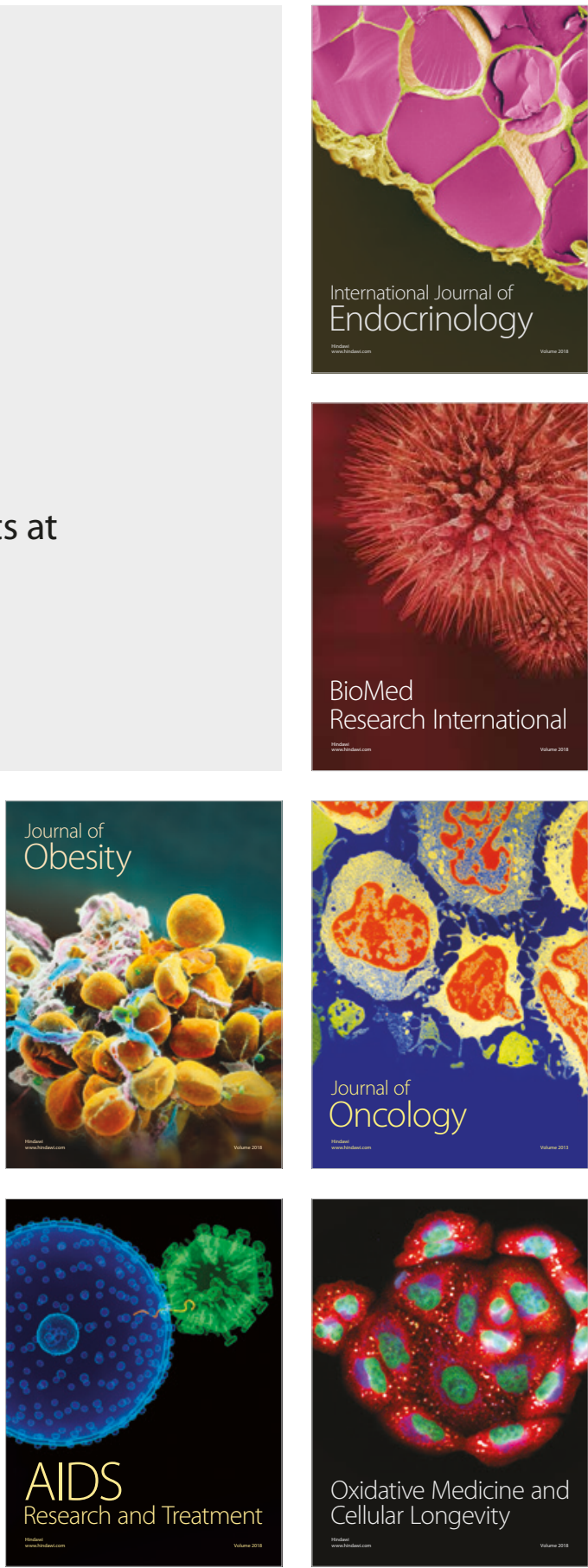